Research Paper

\title{
Discoloration of Provisional Restorations after Oral Rinses
}

\author{
Sedanur Turgut ${ }^{1}$, Bora Bagis², Elif Aydogan Ayaz¹, Kıvanç Utku Ulusoy³, Subutay Han Altintas¹, Fatih \\ Mehmet Korkmaz ${ }^{1}$, Nilsun Bagis ${ }^{凶}$
}

1. DDS, PhD, Department of Prosthodontics, Faculty of Dentistry, Karadeniz Technical University, Trabzon, Turkey;

2. DDS, PhD, Department of Prosthodontics, Faculty of Dentistry, Izmir Katip Celebi University, Izmir, Turkey;

3. DDS, PhD, Department of Prosthodontics, Faculty of Dentistry, Suleyman Demirel University, Isparta, Turkey;

4. DDS, PhD, Department of Periodontology, Faculty of Dentistry, Ankara University, Ankara, Turkey.

$\square$ Corresponding author: Associative Professor Bora Bagis, Phd, DDs. Head of the Department of Prosthodontics, Faculty of Dentistry, Izmir Katip Celebi University, Trabzon, Turkey. Address: Aydınlık Evler Mahallesi, Cem Meriç Caddesi, 6780 Sokak. No: 48, 35640-Çiğli / IZMIR. E-mail: [bbagis@yahoo.com] Telephone: +90 5326804656.

(C) Ivyspring International Publisher. This is an open-access article distributed under the terms of the Creative Commons License (http://creativecommons.org/ licenses/by-nc-nd/3.0/). Reproduction is permitted for personal, noncommercial use, provided that the article is in whole, unmodified, and properly cited.

Received: 2013.05.08; Accepted: 2013.08.21; Published: 2013.08.30

\begin{abstract}
Purpose: Oral rinses are widely used to promote periodontal health with provisional restorations during the interim period. The aim of this study was to compare the discoloration of provisional restoration materials with different oral rinses.

Material and Methods: A total of I 40 disc-shaped specimens (shade A2) $(10 \mathrm{~mm} \times 2 \mathrm{~mm}$ ) were prepared from one PMMA-based (TemDent Classic $^{\circledR}$ ) and three different bis-acrylic-based (Protemp $\|^{\circledR}{ }$, Luxatemp ${ }^{\circledR}$ and Fill- $\left.\mathrm{In}^{\circledR}\right)$ provisional restoration materials $(n=7)$. The color values $\left(L^{*}\right.$, $\mathrm{a}^{*}$, and $\mathrm{b}^{*}$ ) of each specimen were measured before and after exposure with a colorimeter, and the color changes $(\Delta \mathrm{E})$ were calculated according to the CIE L*a*b* system. The specimens were immersed in each of the 4 oral rinses (alcohol-containing mouthwash, chlorhexidine, benzydamine $\mathrm{HCl}$, benzydamine $\mathrm{HCl}$ and chlorhexidine) twice a day for 2 minutes. After 2 minutes of immersion in the oral rinses, the specimens were immersed in artificial saliva. The specimens were exposed to the oral rinses and the artificial saliva for 3 weeks. Two-way ANOVA, the Bonferroni test and the paired sample t-test were used for statistical analyses $(p<0.05)$.

Results: Comparison of the discoloration from the oral rinses after immersion for three weeks revealed no significant differences $(p>0.05)$. The lowest color change was observed in PMMA-based Temdent in all oral rinses $(p<0.05)$. There were no significant differences between the bis-acryl composites after immersion in saliva or the mixture of benzydamine $\mathrm{HCl}$ and chlorhexidine and the alcohol-containing mouthwash for 3 weeks ( $p>0.05)$. After immersion in chlorhexidine, the color change values of Protemp II and Fill-in showed significant differences $(p=0.018)$. Protemp II also showed less discoloration than the other bis-acryl composites, and this color change was statistically significant $(\mathrm{p}<0.05)$. For all oral rinses, the $L^{*}$ value decreased while $b^{*}$ values increased, and this color change was found to be statistically significant $(p<0.05)$. $A^{*}$ values were found to be significantly higher with oral rinses $(p<0.05)$, except Protemp II immersed in benzydamine $\mathrm{HCl}$ or alcohol-containing mouthwash.

Conclusions: The type of the oral rinse did not affect the discoloration process. For long-term esthetic results, choosing MMA-based materials for provisional restorations appears to be more effective.
\end{abstract}

Key words: discoloration, provisional restorations, oral rinses. ('PMMA, Polymethyl methacrylate'; 'HCL, Hydrochloride'; 'CIE, Commission Internationale de l'Eclairage'; MMA, Methyl methacrylate'). 


\section{INTRODUCTION}

Multiple areas of concern have been identified with provisional restorations, including esthetics, periodontal health, maxillomandibular relationships, and continued evaluation of fixed prosthodontic treatment plans. [1] Biologically acceptable fixed prosthetic treatments demand that prepared teeth are protected and stabilized with provisional restorations that resemble the form and function of the definitive treatment. These restorations sometimes serve as a diagnostic aid in treatment assessment, and in cases of temporomandibular joint or periodontal disorders, treatment plans require long-term provisionalization until the definitive prostheses have been fabricated. Therefore, the esthetics of the temporary restoration become a concern, particularly when it is in the esthetic zone during these processes.[2]

In esthetically critical areas, the provisional restoration must provide an initial shade match and maintain an esthetic appearance over the period of service. [3] Discoloration of the restoration may be an esthetic problem, resulting in patient dissatisfaction and additional time and expense for replacement. [4] This is particularly problematic when provisional restorations are subjected to prolonged exposure to colorants during lengthy treatments. [5]

Oral rinses are widely used to promote periodontal health during this interim period, whereas provisional restorations assist in the maintenance of periodontal health and promote guided tissue healing by providing a matrix for the surrounding gingival tissues. Oral rinses used in conjunction with provisional treatment have been shown to reduce plaque levels and to improve gingival health. [6] These rinses are also occasionally administered after tooth preparation because of their anti-inflammatory, antiseptic, and analgesic properties. [7,8] They can affect the color stability of provisional restorations and cause discolorations secondary to dietary factors and medications. Hence, discoloration may be a significant criterion when selecting a particular provisional restoration in an esthetically critical area. [9] Bagis et al. have reported that the use of oral rinses for three weeks caused discoloration of natural teeth and that this discoloration was clinically unacceptable. Currently, the daily use of mouth rinses has become popular, and studies have reported that Listerine, which is an alcohol-containing mouthwash, has results that are comparable to chlorhexidine or benzydamine HCL and has a significant effect on plaque inhibition. [10] However, our knowledge is limited regarding the discoloration effect of these daily used oral rinses.

Many authors have used various staining solu- tions to observe the discoloration capacity of various provisional materials. $[3,5,8]$ Generally, acrylic-based and composite resin-based materials have been used for the fabrication of provisional restorations. As these compositions and curing degrees can differ from each other, there are also differences in the staining characteristics of these materials. It has been reported that acrylic-based materials have a homogeneous composition and that composite-based materials have a more heterogeneous composition. Generally, acrylic-based materials exhibited a better color stability than the composite materials, and the authors attributed this discoloration to the oxidation of unreacted double bonds in the composite material. [11,12]

Oral rinses are widely used during this interim period, and there have been no studies in the literature in which oral rinses were used as discoloration agents for provisional restorations. The aim of this study was to examine the effects of four different oral rinses on the color stability of one acrylic-based and three composite-based provisional restoration materials after immersion for 3 weeks.

The hypothesis of this study was that the discoloration of provisional restorations is related to the type of provisional restoration material and type of oral rinse.

\section{MATERIALS AND METHODS}

Four staining solutions (alcohol-containing mouthwash, chlorhexidine, benzydamine $\mathrm{HCl}$, benzydamine $\mathrm{HCl}$ and chlorhexidine) and four provisional restoration materials (one chemically activated polymethylmethacrylate and three chemically activated bis-acryl composites) were used for this study. The product names, types of materials and manufacturers are listed in Table 1.

\section{Preparation of Specimens}

Thirty-five disc-shaped specimens (shade A2), 10 $\mathrm{mm}$ in diameter and $2 \mathrm{~mm}$ in thickness, were prepared for each provisional material using a brass mold with a socket $(n=7)$, and these specimens were randomly divided into 5 groups for the solutions. The materials were dispensed, manipulated, and polymerized according to the manufacturers' instructions. The chemically cured materials were mixed in a mixing cup according to the manufacturers' suggested ratio, using a glass spatula until a homogeneous mix was obtained according to the manufacturer's directions. The materials were then placed separately into the mold and sandwiched between two glass plates. After completely setting, the excess provisional materials were ground by hand lapping with a 
1000-grit silicon paper for 10 seconds. The surfaces of the specimens were polished by one operator for 15 seconds using pumice, which was followed by rinsing with distilled water to remove any debris before immersion. All of the specimens were kept dry at room temperature until all specimens were fabricated.

\section{Preparation of the Staining Solutions}

Artificial saliva was prepared, and it consisted of the following ingredients: $1 \mathrm{~g}$ sodium carboxymethylcellulose, $4.3 \mathrm{~g}$ xylitol, $0.1 \mathrm{~g}$ potassium chloride, $5 \mathrm{mg}$ calcium chloride, $40 \mathrm{mg}$ potassium phosphate, $1 \mathrm{mg}$ potassium thiocyanate and $100 \mathrm{~g}$ deionized water [13].

Each oral rinse (chlorhexidine, benzydamine $\mathrm{HCl}$, benzydamine $\mathrm{HCl}$ and chlorhexidine, alcohol-containing mouthwash) and the artificial saliva were maintained in a dark environment at $37^{\circ} \mathrm{C} \pm 1^{\circ} \mathrm{C}$ to stimulate the conditions in an oral cavity. The discoloration solutions were changed twice a day throughout the three weeks.

\section{Experimental Procedure}

All of the specimens were stored in artificial saliva at $37^{\circ} \mathrm{C} \pm 1^{\circ} \mathrm{C}$ for 24 hours. The rehydration simulated the first day of service for provisional restorations in the oral environment. It is known that the types of materials used in this study imbibe most of the water during the first day of immersion. $[3,14]$

Before measurements were performed, each specimen was rinsed with distilled water for 30 seconds, gently cleaned with a soft toothbrush and gently dried.

The color values $\left(\mathrm{L}^{*}, \mathrm{a}^{*}, \mathrm{~b}^{*}\right)$ of each specimen were measured before exposure with a colorimeter (Shade Eye Ex; Shofu, Japan) in a viewing booth under a standard illuminant, and the values were recorded according to the CIE $\mathrm{L}^{*} \mathrm{a}^{*} \mathrm{~b}^{*}$ system.

The CIE L*a*b* system is an approximately uniform color space with coordinates for lightness, namely white-black $\left(\mathrm{L}^{*}\right)$, redness-green $\left(\mathrm{a}^{*}\right)$, and yellowness-blueness $\left(b^{*}\right)$. The measurements were repeated 3 times for each specimen, and the mean values of $L^{*}, a^{*}, b^{*}$ were calculated. After the baseline color measurements were obtained, the specimens were immersed into the discoloration solutions. The four restorative material specimens were distributed into 5 groups $(n=7)$. Randomly selected specimens from each material were immersed in each of the 4 staining solutions twice a day for 2 minutes. After 2 minutes of immersion in the oral rinses, the specimens were immersed in artificial saliva. Thus, the specimens were exposed to oral rinses for a total of 84 minutes, which is equivalent to the time of mouth rinse use for 3 weeks. The remaining specimens from each material served as a control group and were stored only in the artificial saliva during the 3 weeks.

After three weeks of immersion, the specimens were rinsed with distilled water for 5 minutes and gently brushed with a soft toothbrush for 15 seconds. At this point, color measurements were recorded with the same colorimeter, and these measurements were performed under the same conditions and in the same manner described for the baseline measurements. The calculation of the color variation $\Delta \mathrm{E}^{*}$ between two color positions (three weeks of storage and baseline) in 3-dimensional $\mathrm{L}^{*} \mathrm{a}^{*} \mathrm{~b}^{*}$ color space is as follows: $\Delta \mathrm{E}^{*}$ $=\left[\left(\mathrm{L}^{*}-\mathrm{L} 0^{*}\right)^{2}+\left(\mathrm{a} 1^{*}-\mathrm{a} 0^{*}\right)^{2}+\left(\mathrm{b} 1^{*}-\mathrm{b} 0^{*}\right)^{2}\right]^{1 / 2}$

\section{Statistical Analyses}

A two-way analysis of variance (ANOVA) was used to evaluate the effects of the material type and discoloration agent on color changes. The mean color change values were compared using the Bonferroni Test. To analyze the $L^{*}, a^{*}, b^{*}$ values of all tested materials, the data were compared using a parametric paired sample t-test. The data analyses were evaluated at a significance level of $p<0.05$ for all individual tests.

Table I. Materials used in the study.

\begin{tabular}{lll}
\hline Product & Material type & Manufacturer \\
\hline Temdent Classic $^{\circledR}$ & Chemically polymerized polymethylmetacrylate (PMMA) & Schütz-Dental Co, Germany \\
Protemp II & Chemically polymerized bis-acryl composite & 3M ESPE, Seefeld, Germany \\
Luxatemp & Chemically polymerized bis-acryl composite resin & DMG, Hamburg, Germany \\
Fill-In ${ }^{\circledast}$ & Chemically polymerized bis-acryl composite & Kerr, De Trey, Germany \\
Klorhex $^{\circledast}$ & $\% 0,12$ Chlorhexidine & Drogsan, Ankara, Turkey \\
Tantum Verde $^{\circledR}$ & $\% 0.15$ Benzidamin HCl & Santa Farma, İstanbul, Turkey \\
Kloroben $^{\circledR}$ & $\% 0.15$ Benzidamin HCl, & Drogsan, Ankara, Turkey \\
& $\% 0.12$ Chlorhexidine & Johnson and Johnson, Turkey \\
\hline Listerin $^{\circledR}$ & Alcohol-containing mouthwash, \%0.064 thymol, \%0.092 eucalyptol, \%0.064 & \\
\hline
\end{tabular}




\section{RESULTS}

The values of the mean color difference $\Delta \mathrm{E}^{*}$ and standard deviations for each combination of discoloration solutions and provisional material are listed in Table 2. The results of the two-way ANOVA and the Bonferroni test indicated that the effects of the interactions between the solutions and the provisional restorations were statistically significant.

After immersion for 3 weeks in saliva or the other oral rinses used in the study, the lowest color change was observed in PMMA-based Temdent ${ }^{\circledR}$ in all oral rinses, which was significantly lower than with the bis-acrylic-based materials $(p<0.05)$. There were no significant differences between the bis-acryl composites after immersion in saliva or the mixture of benzydamine $\mathrm{HCl}$ and chlorhexidine or alcohol-containing mouthwash for 3 weeks ( $p>0.05)$. After immersion in the chlorhexidine solution, the color change values of the bis-acryl-composite-based Protemp II ${ }^{\circledR}$ and Fill-in ${ }^{\circledR}$ showed significant differences $(p=0.018)$. Protemp II ${ }^{\circledR}$ also showed higher dis- coloration than the other bis-acryl composites, and this color change was found to be statistically significant $(\mathrm{p}<0.05)$.

Comparison of discolorations with the oral rinses after immersion for three weeks revealed no significant differences $(p>0.05)$.

The $L^{*}, a^{*}$ and $b^{*}$ values of the tested materials after immersion in saliva and the oral rinses were compared using the paired sample $t$-test. The mean $L^{*}, a^{*}$ and $b^{*}$ values are listed in Tables 3,4 and 5 . The results indicated that there were significant differences between the $L^{*}, a^{*}$ and $b^{*}$ values after immersion in the oral rinses. For all oral rinses, the $\mathrm{L}^{*}$ value decreased while the $b^{*}$ values increased, and this color change was found to be statistically significant $(p$ $<0.05)$. The $a^{*}$ values were found to be significantly higher with oral rinses $(\mathrm{p}<0.05)$, except for Protemp II $^{\circledR}$ immersed in benzydamine $\mathrm{HCl}$ and Temdent ${ }^{\circledR}$ immersed in benzydamine $\mathrm{HCl}$ and in the alcohol-containing mouthwash.

Table 2. Mean and SD values of color changes after immersion of 3 weeks.

\begin{tabular}{|c|c|c|c|c|c|}
\hline & Saliva & Chlorhexidine & BenzidaminHCl & $\begin{array}{l}\text { Benzidamin } \mathrm{HCl} \text {, } \\
\text { Chlorhexidine }\end{array}$ & $\begin{array}{l}\text { Alcohol-containing } \\
\text { mouthwash }\end{array}$ \\
\hline Temdent ${ }^{\circledR}$ & $1,94 \pm 0,4^{\text {(a) }}$ & $3,52 \pm 0,2$ (a) & $3,35 \pm 0,6$ (a) & $3,04 \pm 0,1$ (a) & $3,02 \pm 0,6$ (a) \\
\hline Protemp II ${ }^{\circledR}$ & $2,73 \pm 0,6$ (b) & $5,38 \pm 0,3$ (a) & $5,17 \pm 0,4(b)$ & $4,94 \pm 0,3(b)$ & $4,87 \pm 0,4(b)$ \\
\hline Luxatemp ${ }^{\circledR}$ & $2,75 \pm 0,2$ (b) & $5,1 \pm 0,1$ (bc) & $4,78 \pm 0,3$ (c) & $5,06 \pm 0,4(b)$ & $4,68 \pm 0,6(b)$ \\
\hline Fill-in ${ }^{\circledR}$ & $2,5 \pm 0,2^{(b)}$ & $4,97 \pm 0,3$ (c) & $4,85 \pm 0,5$ (c) & $4,93 \pm 0,3{ }^{(b)}$ & $4,8 \pm 0,3$ (b) \\
\hline
\end{tabular}

Same letters in the same columns were not significantly different (Bonferroni test $(\mathrm{p}<0.05)$.

Table 3. Mean and SD values of $L^{*}$ values after immersion of 3 weeks.

\begin{tabular}{llllll}
\hline & Saliva & Chlorhexidine & BenzidaminHCl & $\begin{array}{l}\text { BenzidaminHCl, } \\
\text { Chlorhexidine }\end{array}$ & $\begin{array}{l}\text { Alcohol-containing mouth- } \\
\text { wash }\end{array}$ \\
\hline Temdent ${ }^{\circledR}$ & $82,9 \pm 0,8$ & $82,3 \pm 0,6^{*}$ & $81,6 \pm 0,5^{*}$ & $82,1 \pm 0,9^{*}$ & $81,5 \pm 0,7^{*}$ \\
Protemp II® & $80,7 \pm 0,6$ & $79,7 \pm 0,8^{*}$ & $79,4 \pm 0,5^{*}$ & $78,4 \pm 0,8^{*}$ & $79,2 \pm 0,6^{*}$ \\
Luxatemp ${ }^{\circledR}$ & $83,4 \pm 1,2$ & $82,2 \pm 0,3^{*}$ & $82,4 \pm 0,7^{*}$ & $82 \pm 0,4^{*}$ & $82,6 \pm 0,5^{*}$ \\
Fill-in ${ }^{*}$ & $78,7 \pm 0,7$ & $76,7 \pm 0,4^{*}$ & $77 \pm 0,6^{*}$ & $77,1 \pm 0,8^{*}$ & $77,3 \pm 0,6^{*}$ \\
\hline
\end{tabular}

Symbol $\left(^{*}\right)$ represents that there were significant differences between the $\mathrm{L}^{*}$ values of tested materials among the tested materials immersed in saliva and the oral rinses.(Paired-Sample T-test $(\mathrm{p}<0.05)$.

Table 4. Mean and SD values of a* values after immersion of 3 weeks.

\begin{tabular}{lllll} 
& Saliva & Chlorhexidine & BenzidaminHCl & \multicolumn{1}{c}{$\begin{array}{l}\text { BenzidaminHCl, } \\
\text { Chlorhexidine }\end{array}$} \\
\hline Temdent $^{\circledR}$ & $1,3 \pm 0,3$ & $1,6 \pm 0,3^{*}$ & $1,3 \pm 0,2$ & $1,4 \pm 0,3^{*}$ \\
Protemp II $^{\circledR}$ & $1,1 \pm 0,1$ & $1,2 \pm 0,2^{*}$ & $1,1 \pm 0,1$ & $1,2 \pm 0,2^{*}$ \\
Luxatemp $^{\circledR}$ & $0,8 \pm 0,2$ & $1 \pm 0,2^{*}$ & $1,2 \pm 0,1^{*}$ & $1,1 \pm 0,1^{*}$ \\
Fill-in $^{\circledR}$ & $1 \pm 0,2$ & $1,4 \pm 0,2^{*}$ & $1,2 \pm 0,2^{*}$ & $1,2 \pm 0,2^{*}$ \\
\hline
\end{tabular}

Symbol $\left(^{*}\right)$ represents that there were significant differences between the $\mathrm{a}^{*}$ values of tested materials among the tested materials immersed in saliva and the oral rinses. (Paired-Sample T-test $(\mathrm{p}<0.05)$. 
Table 5. Mean and SD values of $b^{*}$ values after immersion of 3 weeks.

\begin{tabular}{llllll}
\hline & Saliva & Chlorhexidine & BenzidaminHCl & $\begin{array}{l}\text { BenzidaminHCl, } \\
\text { Chlorhexidine }\end{array}$ & $\begin{array}{l}\text { Alcohol-containing } \\
\text { mouthwash }\end{array}$ \\
\hline Temdent $^{\circledR}$ & $14,9 \pm 0,7$ & $19,6 \pm 0,6^{*}$ & $19,1 \pm 0,7^{*}$ & $16,8 \pm 0,3^{*}$ & $16,5 \pm 0,5^{*}$ \\
Protemp II $^{\circledR}$ & $15,2 \pm 0,6$ & $16,2 \pm 0,6^{*}$ & $15,8 \pm 0,5^{*}$ & $16,2 \pm 0,6^{*}$ & $16,7 \pm 0,4^{*}$ \\
Luxatemp $^{\circledR}$ & $16,8 \pm 0,9$ & $18,6 \pm 0,4^{*}$ & $18,1 \pm 0,6^{*}$ & $18,4 \pm 0,8^{*}$ & $18 \pm 0,8^{*}$ \\
Fill-in $^{\circledR}$ & $17,9 \pm 1,1$ & $19,6 \pm 0,8^{*}$ & $18,3 \pm 0,8^{*}$ & $19,1 \pm 0,8^{*}$ & $18,7 \pm 0,9^{*}$ \\
\hline
\end{tabular}

Symbol $(*)$ represents that there were significant differences between the $b^{*}$ values of tested materials among the tested materials immersed in saliva and the oral rinses. (Paired-Sample T-test $(\mathrm{p}<0.05)$.

\section{DISCUSSION}

The working hypothesis of the present study should be partially accepted because there were significant differences in discoloration depending on the type of provisional material used and because the oral rinses affected the color stability of these materials after immersion for three weeks. However, the discoloration of the provisional restoration materials was not affected by the type of oral rinse.

According to the results of this study, PMMA-based provisional materials provide more color stability than the bis-acryl composite-based materials. These results may be due to the homogeneous composition of the acrylic-based material and the heterogeneous composition of the composite material. The discoloration might be due to both surface adsorption and absorption of the colorants of the oral rinses. Fine colorant particles may have deposited in the pits of the bis-acryl composites. The less polar colorants and water-soluble polyphenols in the colorants may have penetrated deep into the materials, possibly because such colorants are more compatible with the polymer matrix of the composites than with methylmethacrylate. [15] The findings of this study are in agreement with a study by Doray et al. [2], who stated that MMA provided better color stability. Additionally, Yannikakis et al. [16] have reported that after immersion of provisional materials in various staining solutions for up to 1 month, the MMA materials exhibited the best color stability and the bis-acryl composite materials exhibited the worst color stability. These chemical discolorations have been attributed to an oxidation of the polymer matrix or oxidation of unreacted double bonds. [8,9] Additionally, the composite-based resins can absorb water at a higher rate because of a high diffusion coefficient in comparison to MMA-based resins. These properties may explain the excessive color changes observed with composite-based materials with the use of oral rinses. [17] Further investigation is needed to evaluate the relationship between the discoloration solution and provisional restorative materials with respect to the chemical properties of these solutions.
The discoloration potential of chlorhexidine gluconate has already been reported in the literature. $[7,18]$ This study confirmed these reports and demonstrated that chlorhexidine-containing mouth rinses discolored the provisional restorative materials more than the controls. However, chlorhexidine gluconate, benzydamine hydrochloride and the effects of the hybrid solution were not significantly different from each other. Listerine, composed of a mixture of essential oils, also leads to discoloration in provisional restoration materials. It may be possible that these restorative materials have a tendency to show discoloration after three weeks, regardless of which oral rinse is being used.

Finishing and polishing procedures may also influence surface smoothness, which is related to early discoloration. Rough surfaces mechanically retain surface stains better than smooth surfaces. [8] Therefore, we used a ground and polished surface, and all the specimens' surfaces were standardized because it is usually necessary to remove excess material after the fabrication of the provisional restorations. It has been reported that MMA-based provisional materials exhibit smoother surfaces after initial polishing methods when compared with bis-acryl-based materials. The significant discoloration of bis-acrylic-based provisional materials in the present study may be attributed to their greater surface roughness. [8]

In the present study, artificial saliva was used to deposit a pellicle layer. Saliva and the subsequent accumulation of pellicles act as a matrix for the deposition of stains, which may result in discoloration. However, this technique was practically different from clinical conditions as salivary pellicle developed without being impacted by chromogens or other agents, such as dietary acid, all of which would affect the final layer. $[7,18]$ These differences from clinical conditions should be considered when extrapolating the results of the present study.

Color changes that are perceptible may compromise the clinical acceptability of a provisional restoration. The value of $\Delta \mathrm{E}^{*}$ represents the numerical distance between the $L^{*} a^{*} b^{*}$ coordinates and the relative color changes that an observer might report for materials. When the $\Delta \mathrm{E}$ value of two colors is 0 , the 
color difference is described as "perfect"; a value of 0.5 to 1.5 units is "very good"; 1 to 2 is "good"; 2 to 3.5 is "clinically perceptible"; and $>3.5$ is unacceptable. [19] In our study, discolorations below or above $\Delta \mathrm{E}^{*}=3.5$ were referred to as 'acceptable' or 'unacceptable', respectively.

According to the results of the present study, the control groups for all materials used exhibited color changes between 1.94-2.5, and this color change was 'good' for MMA-based material and 'clinically perceptible' for bis-acryl-based materials. MMA-based provisional materials showed $\Delta \mathrm{E}^{*}$ values lower than 3.5 for all solutions, and this color change was 'clinically perceptible'. In contrast, bis-acryl-based provisional restorations showed higher values and were deemed 'clinically unacceptable'.

Therefore, if provisional restorations are being used for a long period of time, MMA-based provisional materials should be chosen. A number of studies have investigated the discoloration of both acrylic and bis-acryl materials under a variety of conditions, including using staining solutions or accelerated aging with ultraviolet (UV) light irradiation. $[8,13,20]$ The results from these studies suggest that the acrylic resin provisional materials tend to be more resistant to changes in color when subjected to discoloration through immersion in solution, whereas the bis-acryl composite resins tend to be more resistant to discoloration when exposed to UV light irradiation. These findings are most likely related to the mode of discoloration in the two types of conditions; in cyclic immersion through a staining solution, discoloration occurs through sorption processes, whereas discoloration from UV light sources occurs through a bulk deterioration process. In the present study, the oral rinses caused all of the provisional restoration materials to become darker, more yellow and red, regardless of the material type. There is limited published data regarding how oral rinses affect the PMMA or composite-based materials. The solutions employed in this study do not include all substances to which provisional materials may be exposed. Although artificial saliva was used in the present study to stimulate conditions in the oral cavity, other factors that could influence the degree of total color change, including thermal cycling, diet or abrasion, were not included. These factors should be considered for future studies.

Color stability is only one variable that must be considered when choosing a provisional material, but it may be of great importance to patients and clinicians when working in the esthetic zone. The discoloration effect of the oral rinses used during this interim period is evident. This interim period sometimes requires long provisionalization, and the patients may wait for 3 weeks or longer until their de- finitive prostheses are fabricated. As the type of the oral rinse did not affect the discoloration process, choosing the best provisional restoration material appears to be more important for long-term esthetic results.

\section{CONCLUSION}

Within the limitations of the study, the following conclusions were drawn:

- All the bis-acryl resins and PMMA-based provisional materials exhibited statistically significant color changes after exposure to oral rinses for 3 weeks.

- There were no significant differences between the discoloration effects of alcohol-containing mouthwash, chlorhexidine, benzydamine $\mathrm{HCl}$, or the benzydamine $\mathrm{HCl}$ and chlorhexidine mixture on the provisional materials.

- Bis-acryl-based provisional materials exhibited more discoloration than the PMMA-based materials, and this discoloration was clinically perceptible.

The provisional materials became darker, yellowish and reddish after immersion, regardless of the type of oral rinse.

\section{Competing Interests}

The authors have declared that no competing interest exists.

\section{References}

1. Rosenstiel SF, Land M, Fujimoto J. Contemporary fixed prosthodontics. 3rd ed. St Louis: Mosby. 2000;: 380-416.

2. Doray PG, Li D, Powers JM. Color stability of provisional restorative materials after accelerated aging. J Prosthodont 2001;: 212-216.

3. Guler AU, Yilmaz F, Kulunk T, Guler A, Kurt S. Effects of different drinks on stainability of resin composite provisional restorative materials. J Prosthet Dent. 2005 Aug;94(2):118-24.

4. Dory PG, Wang X, Powers JM, Burgess J. Accelerated Aging Affects Color Stability of Provisional Restorative Materials. J Prosthod. 1997; 6:183-188.

5. Sham AS, Chu FC, Chai J, Chow TW. Color stability of provisional prosthodontic materials. J Prosthet Dent. 2004; 91:447-52.

6. Sorensen JA, Doherty FM, Newman MG, Flemmig TF. Gingival enhancement in fixed prosthodontics. Part I: Clinical findings. J Prosthet Dent 1991; 65:100-7.

7. Bagis B, Baltacioglu E, Özcan M, Ustaomer S. Evaluation of chlorhexidine gluconate mouthrinse-induced staining using a digital colorimeter: an in vivo study. Quintessence Int. 2011; 42:213-23.

8. Yannikakis SA, Zissis AJ, Polyzois GL, Caroni C. Color stability of provisional resin restorative materials. J Prosthet Dent. 1998; 80:533-9.

9. Doray PG, Li D, Powers JM. Color stability of provisional restorative materials after accelerated aging. J Prosthodont 2001; 10:212-6.

10. Fine DH. Listerine: past, present and future- Atest of thyme. J Dent 2010; 38:2-5.

11. Geurtsen W. Substances released from dental resin composites and glass ionomer cements. Eur J Oral Sci. 1998; 106:687-95.

12. Haselton DR, Arnold MD, Dawson DV. Effect of storage solution on surface roughness of provisional crown and fixed partial denture materials. J Prosthodont 2004; 13:227-232.

13. Yumiko HY, Watanabe E, Tadokoro K, Inoue T, Miyazaki M, Franklin R. Effects of ammonium hexafluorosilicate application on demineralized enamel and dentin of primary teeth. Oral Sci. 2012; 54:267-272. 
14. Khan Z, Razavi R, Von Fraunhofer JA. The physical properties of a visible light-cured temporary fixed partial denture material . J Prosthet Dent . 1988; 60:543-5.

15. Haselton DR, Arnold DM, Dawson DV. Color stability of provisional crown and fixed partial denture resins. J Prosthet Dent. 2005; 93:70-5.

16. Sham AS, Chu FC, Chai J, Chow TW. Color stability of provisional prosthodontic materials. J Prosthet Dent. 2004; 91:447-52.

17. Bernardi F, Pincelli MR, Carloni S, Gatto MR, Montebugnoli L. Chlorhexidine with an antidiscoloration system. A comparative study. Int J Dent Hyg. 2004;2:122-126.

18. Carpenter GH., Pramanik R., Proctor GB. An invitro model of chlorhexidine induced tooth staining. J Periodontal Res 2005; 40:225-230.

19. O'Brien WJ. Dental Materials and Their Selection. 3rd ed. Chicago: Quintessence Publishing Co Inc. 2002: 25-38.

20. Givens EJ, Neiva G, Yaman P, Dennison JB. Marginal adaptation and color stability of four provisional materials. J Prosthodont 2008; 17:97-101. 